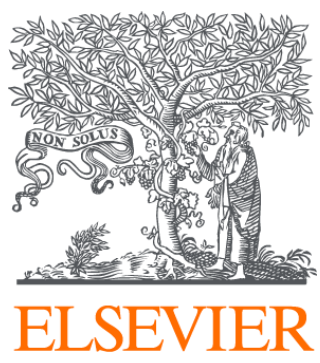

Since January 2020 Elsevier has created a COVID-19 resource centre with free information in English and Mandarin on the novel coronavirus COVID-

19. The COVID-19 resource centre is hosted on Elsevier Connect, the company's public news and information website.

Elsevier hereby grants permission to make all its COVID-19-related research that is available on the COVID-19 resource centre - including this research content - immediately available in PubMed Central and other publicly funded repositories, such as the WHO COVID database with rights for unrestricted research re-use and analyses in any form or by any means with acknowledgement of the original source. These permissions are granted for free by Elsevier for as long as the COVID-19 resource centre remains active. 


\title{
Evaluation and Management of Pediatric Neck Masses
}

\section{An Otolaryngology Perspective}

\author{
Denise L. Jackson, PA-C, MA, CCC-SLP
}

\section{KEYWORDS}

- Cervical fascial spaces • Embryology • Congenital • Ectopic tissue

- Vascular malformations • Pediatric neck mass • Lymphadenopathy

\section{KEY POINTS}

- A comprehensive knowledge of the borders and contents of the fascial spaces of the neck is essential to the proper evaluation and diagnosis of pediatric neck lesions.

- Cervical neck spaces are characterized as being anterior or posterior triangle, central or lateral, and by level of the neck.

- Most pediatric neck masses can be characterized as congenital, inflammatory, infectious, or neoplastic (benign or malignant).

- The 2 most common congenital neck lesions in children are branchial cleft and thyroglossal duct anomalies.

- Infectious neck masses can be described as acute or chronic, and range in etiology from common viral exanthems to tick-borne illnesses.

\section{INTRODUCTION}

Generally, neck masses in children fall into 1 of 3 categories: congenital, inflammatory, or neoplastic. Although malignancies do occur, most neck masses in children are benign in nature. The objective of this article is to provide practitioners guidance in performing comprehensive physical examinations of the pediatric neck mass, clinical decision making and pursuit of pertinent testing, recognizing diagnostic criteria for various entities, and awareness of appropriate treatment plans.

\section{HISTORY}

The age of the child at onset and the duration of the mass are both significant diagnostic factors when taking a history. Knowledge of prior or recent infections of the head and

Disclosure Statement: The author has nothing to disclose.

Otolaryngology-Head and Neck Surgery, University of Virginia Medical Center, 1 Hospital Drive, 2nd Floor, OMS, Room 2741, Charlottesville, VA 22903, USA

E-mail address: DJ7Z@hscmail.mcc.virginia.edu 
neck is important. History of a known tick bite prompts suspicion for tick-borne illnesses in a child with a neck mass, with the specific illness depending on geographic region of residence. Bartonella henselae or toxoplasmosis play an important role in human pathogenicity, and should be considered if there has been exposure to a cat or cat feces. For those with a history of unpasteurized milk product ingestion or contaminated soil exposure, those from endemic regions, those with immunocompromised state, and/or a history of tuberculosis (TB) exposure, etiologic considerations would include atypical mycobacteria, TB, or human immunodeficiency virus (HIV), respectively. A patient history of head or neck radiation should be considered. Family history is also key, because disorders with a clear hereditary pattern such as multiple endocrine neoplasia syndrome type 2, neurofibromatosis, head and neck cancers, autoimmune disorders, or vascular anomalies may be linked to the patient's presenting neck lesion.

\section{PHYSICAL EXAMINATION AND MASS IDENTIFICATION Mass Location}

A comprehensive knowledge of the borders and contents of the fascial spaces of the neck is essential in diagnosing and characterizing pediatric neck lesions. ${ }^{1}$ The neck may be divided into central and lateral spaces. The lateral neck is further divided into the anterior triangle and the posterior triangle. Neck lesions are also described according to levels ranging from IA to VI (Fig. 1). ${ }^{2}$

The central neck comprises dissection levels IA and VI. Its borders extend midline from the mentum to the sternal notch, with palpable structures including the hyoid bone, thyroid and cricoid cartilage, thyroid gland, and upper trachea. ${ }^{1}$ The lateral neck is divided into anterior and posterior triangles, delineated by the sternocleidomastoid (SCM) muscle. The anterior triangle, including neck dissection levels IB to IV, ranges from anterior to

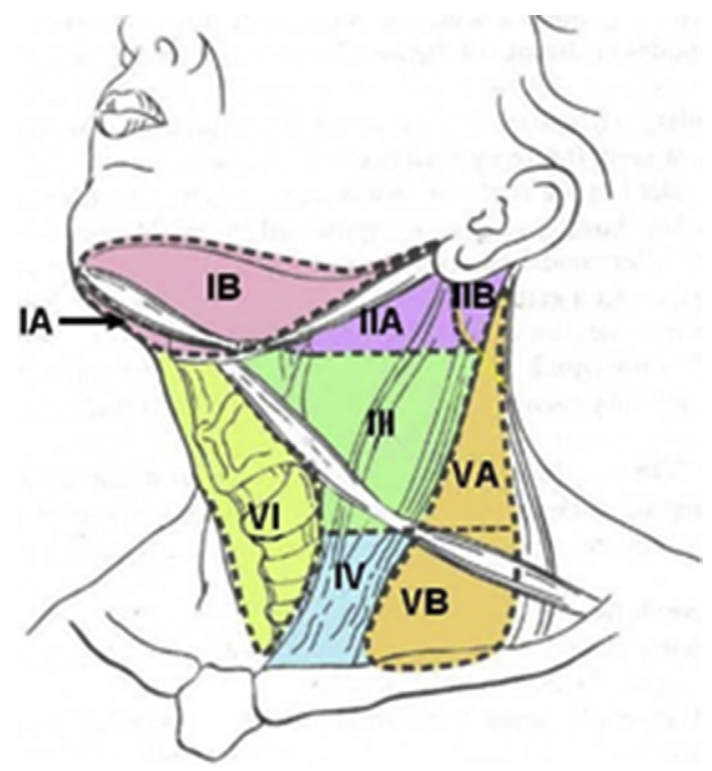

Fig. 1. Neck dissections: radical to conservative. The central neck comprises dissection levels IA (pink) and VI (yellow). The anterior triangle, including neck dissection levels IB to IV (pink, purple, green, and blue). From the posterior border of the SCM to thetrapezius muscle and inferiorly to the clavicle (orange). (From Harish K. Neck dissections: radical to conservative. World J Surg Oncol 2005;3(1):21; with permission.) 
the posterior border of the SCM to midline, superiorly along the inferior border of the mandible to the mastoid tip, and inferiorly to the clavicle. ${ }^{1}$ The posterior triangle runs from the posterior border of the SCM to the trapezius muscle and inferiorly to the clavicle. ${ }^{1}$ Lesions in the supraclavicular fossa have a much higher likelihood of malignancy. ${ }^{1}$

In describing the quality of a neck mass, specific characteristics to include are the size, mobility, tenderness, and appearance of the overlying skin. Benign characteristics include mobility within the soft tissue plane (rather than fixed), well-circumscribed, and small in size. It is important to know if any illness or trauma preceded the mass. Does the lesion fluctuate in size and, if so, what prompts these changes? Drainage or pitting would suggest congenital fistula or deep abscess. If a midline mass elevates with swallowing or tongue protrusion, a thyroglossal duct cyst (TDC) would be likely. In pediatric patients, biopsy is more frequently pursued in larger lesions that persist several weeks, do not resolve with antibiotic therapy, are fixed, or are in the supraclavicular fossa.

\section{Comprehensive Head and Neck Examination}

In addition to describing mass location and quality, a complete head and neck examination is crucial, including assessments of regional skin, the ears, the nose, and the oral cavity. The skin on the head and neck, including the scalp, is important to assess because insect bites, evidence of trauma, or dermatologic conditions may be identified as the source of lymphadenopathy. Examination of the oral cavity may reveal mucosal or dental lesions that may also contribute to formation of neck masses. Meticulous neck examination, beyond the mass itself, including thyroid inspection and palpation, is fundamental. A gross cranial nerve assessment should also be performed. Flexible nasopharyngolaryngoscopy may be indicated in some cases.

\section{DIAGNOSTIC TESTING Laboratory Tests}

Laboratory tests to consider for the workup of acute lymphadenitis include monospot or serology for Epstein-Barr virus and cytomegalovirus, and/or phlebotomy to include a complete blood count with differential, at a minimum. It is appropriate to culture any material expressed from an abscess or from any surface lesion, that is, a nasaopharyngeal culture for increasingly more common respiratory viruses such as metapneumovirus and respiratory syncytial virus, or tonsillar culture if group $A$ streptococcus is suspected. $A B$ henselae immunofluorescence assay blood test assesses for cat scratch disease if there is a history of such an incident or exposure. Toxoplasma gondii antibodies (immunoglobulins $\mathrm{G}$ and $\mathrm{M}$ ) should be considered if applicable, as well as Lyme and ehrlichiosis titers, HIV testing, tuberculin skin testing, and a chest radiograph, if the TB test is positive. Acidfast bacilli testing is important in confirming atypical mycobacteria. If thyroid involvement is probable, a thyroid panel may be considered. Further workup largely depends on the location and characteristics of a lesion. If an abscess is present, fine needle aspiration with a Gram stain would be recommended, to facilitate directed antibiotic therapy, along with acid-fast bacilli staining for mycobacteria, if suspected. Flexible nasopharyngolaryngoscopy will often be deemed necessary for upper airway evaluation. If neoplasm is present, laboratory tests and excisional biopsy are confirmatory of diagnosis, and appropriate referrals will need to be made for multidisciplinary management.

\section{Imaging}

Various imaging modalities, including ultrasound examination, computed tomography (CT) scans, MRI, and MR angiography are used for the evaluation of pediatric neck lesions, and each has its own advantages, limitations, and indications. ${ }^{3}$ The radiologic 
evaluation of pediatric patients after physical examination often begins with conventional ultrasound examination and color Doppler ultrasound examination owing to its nonionizing and noninvasive ability to depict superficial structures. ${ }^{3}$ Ultrasound examination can define the size and extent of localized superficial masses and determine a cystic or solid nature. Color Doppler ultrasound examination can demonstrate the vascularization of the mass, displaced normal surrounding vessels, or intralesional flow. ${ }^{3}$ However, ultrasound examination findings may not allow definitive characterization, especially for deeper lesions. ${ }^{3}$ CT scanning aids in the morphologic characterization and staging of neck masses, and allows for the precise visualization of fine bone structures, calcifications, and deep soft tissue compartments that cannot be demonstrated with ultrasound examination. ${ }^{3}$ Particular attention should be paid to minimizing the radiation exposure of CT scans, particularly in pediatric patients. ${ }^{3} \mathrm{MRI}$, with an absence of ionizing radiation and multiplanar capability, offers superior contrast resolution in evaluating masses in complex areas, including the head and neck. ${ }^{3}$ CT scanning is a more rapid study than $\mathrm{MRI}$, but either modality may require intravenous sedation for younger patients. ${ }^{3}$

The gold standard for diagnosis of vascular lesions involves MRI with gadolinium and/or Doppler ultrasound examination to measure flow. ${ }^{4}$ Arteriovenous malformation and arteriovenous fistula are high-flow lesions, whereas venous and lymphatic malformations are low-flow lesions. ${ }^{5}$ Both hemangiomas and vascular anomalies enhance on T2-weighted images. ${ }^{1}$ Lymphatic malformations appear as multilocular cystic lesions on ultrasound imaging, and also show hyperintensity on T2-weighted MRI. ${ }^{4}$ For embryologic tracts, MRI with fistulogram helps to determine the extent and final disposition of the tract. Each case warrants careful consideration regarding imaging so as to obtain the greatest yield with the least risk to the patient.

\section{EMBRYOLOGY}

\section{Branchial Apparatus}

A comprehensive review of head and neck embryology is beyond the scope of this article. However, a brief review of the embryologic pathway for the branchial apparatus, which is determined in the first 8 weeks in utero, informs our understanding of congenital neck masses. ${ }^{6}$ The branchial apparatus consists of paired pharyngeal arches, pharyngeal pouches, pharyngeal clefts (or grooves), and pharyngeal membranes. ${ }^{6}$ Pharyngeal arches are paired structures that contribute to the formation of the face, jaw, ear, and neck. ${ }^{6}$ The first pharyngeal arch appears around the beginning of the fourth week and others are sequentially added more caudally, with 5 arches present by the end of the fourth gestational week; the fifth arch halts in development, such that the remaining arches are numbered $1,2,3,4$, and $6 .{ }^{6}$ The pharyngeal apparatus forms infoldings or pouches between the arches. ${ }^{6}$ Externally, the pharyngeal apparatus forms outer pharyngeal clefts (or grooves). ${ }^{6}$ Anomalies of the branchial apparatus can present as fistulas (incomplete closure of pouches and clefts, resulting in communication between 2 body surfaces), cysts (trapped embryologic remnants with no external communication), and sinuses (incomplete closure of pouches and clefts, with single body surface communication involving skin or pharynx). ${ }^{1}$ Specific types of branchial apparatus anomalies are highlighted elsewhere in the article.

\section{CONGENITAL NECK MASSES \\ Central Congenital Neck Masses}

\section{Thyroglossal duct cysts}

TDCs are the most common congenital neck masses. They are midline lesions that arise as the result of a residual tract left by the thyroid gland during its embryologic 
descent from the foramen cecum at the base of the tongue, downward to its definitive position $^{1}$ (Figs. 2-4). TDCs characteristically elevate with tongue protrusion or swallowing. ${ }^{1}$ They are very well-circumscribed, mobile, and nontender. Often, TDCs go unrecognized until they enlarge in response to an infection; therefore, the most common age of presentation is in young, school-aged children. ${ }^{1} \mathrm{MRI}$ or CT scans can delineate the full extent of the lesion for perioperative planning; however, ultrasound imaging may be sufficient for radiologic assessment of a central neck mass with a high suspicion for TDC. ${ }^{1}$ Confirmation of normally functional thyroid tissue before TDC resection is also accomplished through preoperative imaging. Once confirmed, a Sistrunk procedure is typically performed, which involves surgical resection of the cystic lesion, along with the entire tract of embryologic descent, up to and including the tract insertion onto a portion of the hyoid bone, thus drastically reducing the rate of TDC recurrence. ${ }^{1}$ This type of surgical intervention is curative in the majority of patients.

\section{Dermoid and epidermoid cysts}

In contrast with TDCs, dermoid and epidermoid cysts are midline lesions (Fig. 5), which do not typically elevate with swallowing or tongue protrusion. Like TDCs, dermoid and epidermoid cysts are characteristically well-circumscribed and typically nontender. These cysts are extremely mobile, and superficial to underlying structures. Dermoid cysts are lined by epithelium and differ from epidermoid cysts in that they contain skin appendages such as sebaceous glands and hair follicles within the cyst wall. ${ }^{4}$ Dermoids may present in later childhood owing to their slow evolution. Forty percent of dermoid and epidermoid cysts are diagnosed at birth, with the majority of the remaining $60 \%$ of these cysts presenting before the age of 5 years. ${ }^{1}$ On noncontrast CT, a dermoid cyst usually appears as a low-density, unilocular,

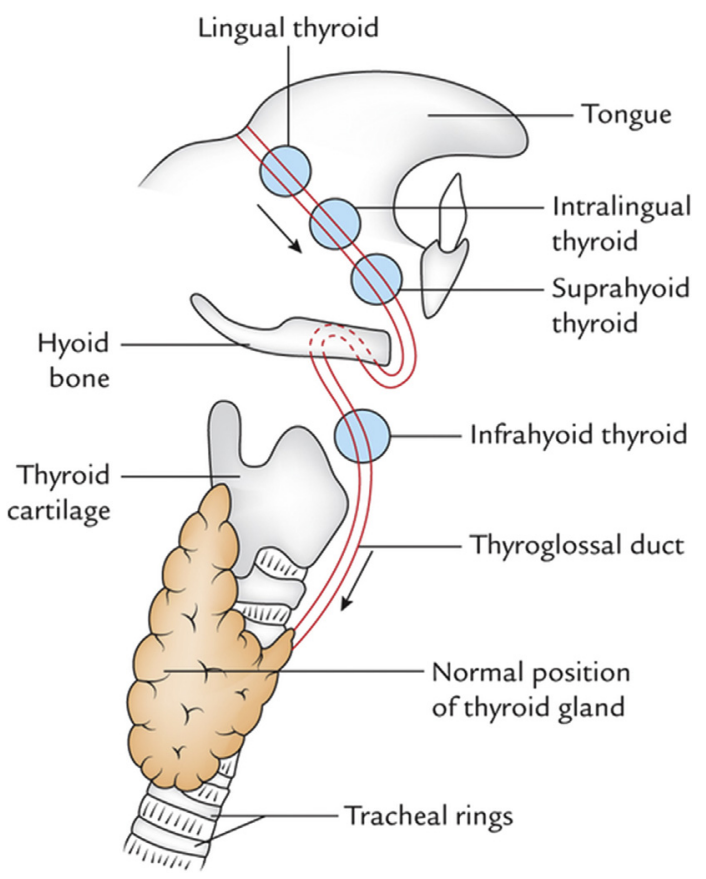

Fig. 2. Path of thyroglossal duct. Note the possible locations of thyroid tissue and thyroglossal cysts in this path. (From Coward K, Wells D, editors. Textbook of clinical embryology. Philadelphia: Elsevier; 2012. p. 122-9; with permission.) 


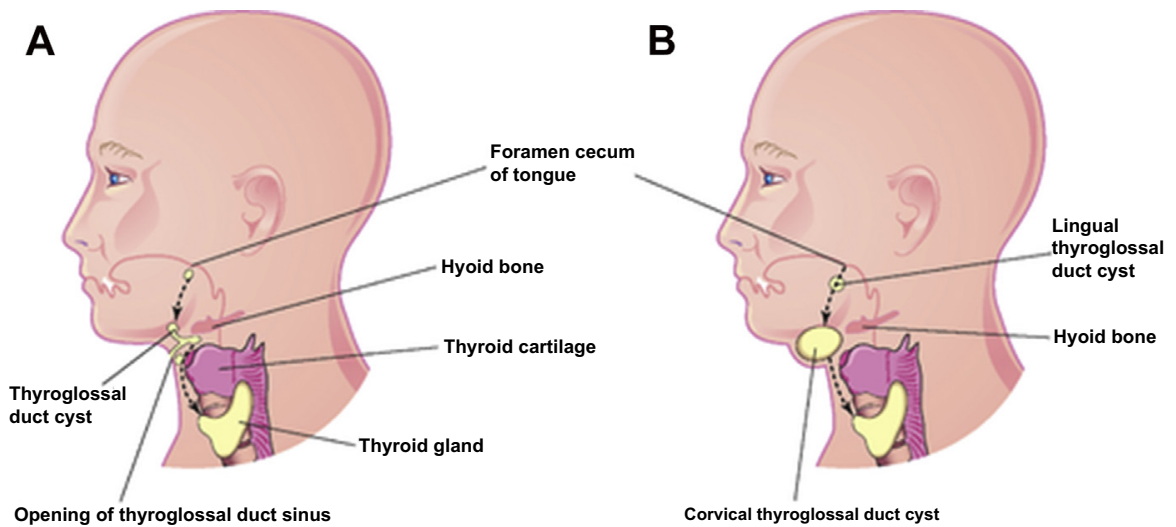

Fig. 3. Arteriovenous malformation of the lower lip. (A) Opening of thyroglossal duct sinus. (B) Cervical thyroglossal duct cyst. (From Coward K, Wells D, editors. Textbook of clinical embryology. Philadelphia: Elsevier; 2012. p. 122-9; with permission.)

well-circumscribed mass. ${ }^{4}$ Fat, mixed-density fluid, and calcification $(<50 \%)$ may also be seen, and there may be coalescence of fat into small nodules within the cystic lesion, giving a "sack of marbles" appearance (Fig. 6). ${ }^{4}$ The presence of calcifications and cystic spaces in these lesions aids in their differentiation from lipomas. ${ }^{4}$ Patients respond well to surgical excision, which is typically curative.

\section{Plunging ranula}

Plunging ranulas may be either congenital or acquired lesions. Their pathophysiology involves obstruction of a sublingual gland, which forms a pseudocyst or mucocele along the floor of the mouth. This cyst becomes termed a "plunging ranula" when the lesion extends into the neck through the mylohyoid ${ }^{1}$ (Fig. 7). Preoperative imaging

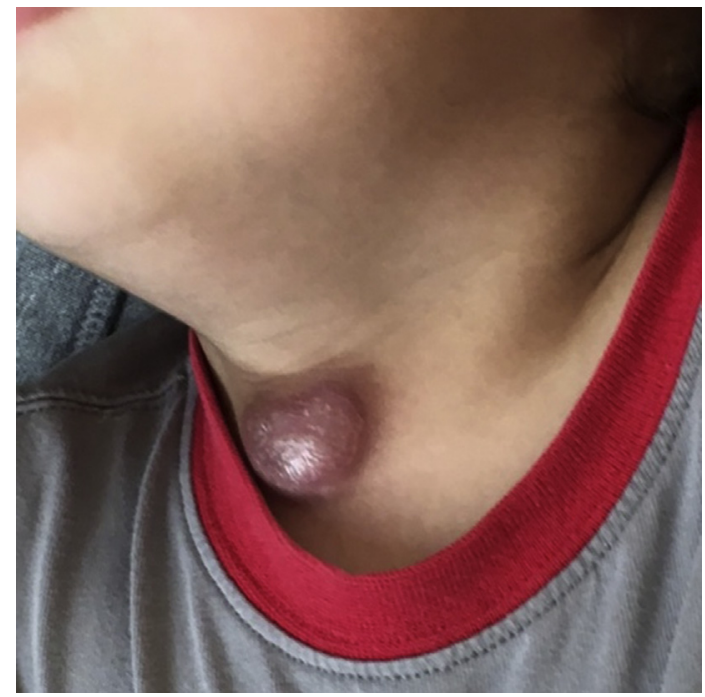

Fig. 4. A 4-year-old boy with a thyroglossal duct cyst. 


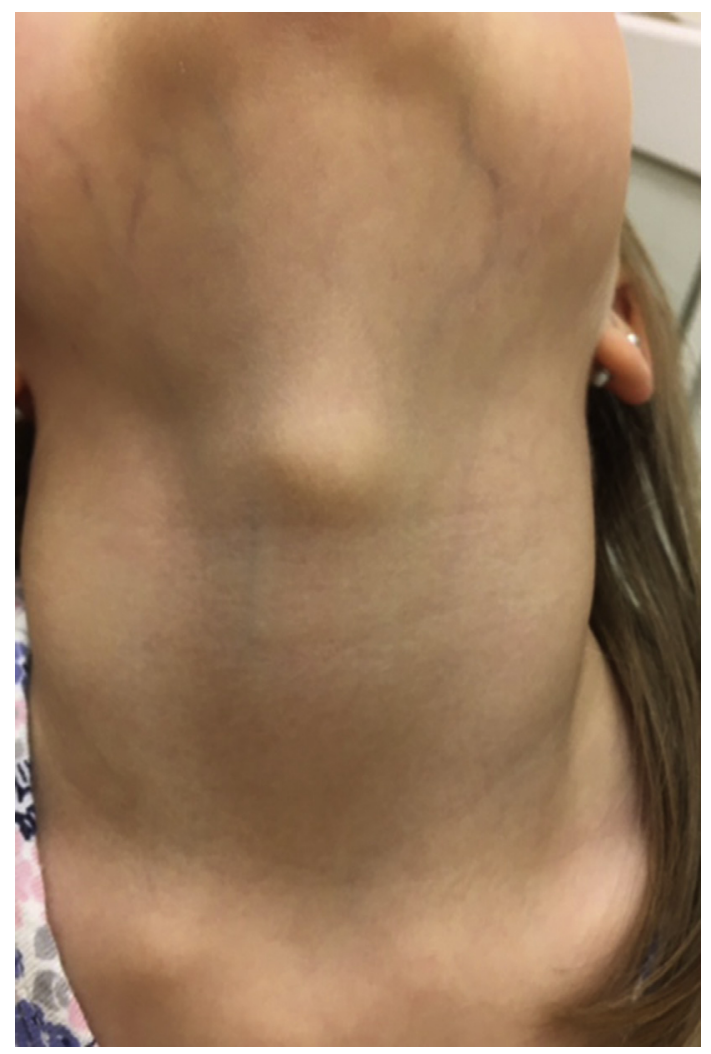

Fig. 5. A 5-year-old girl with an epidermal cyst.

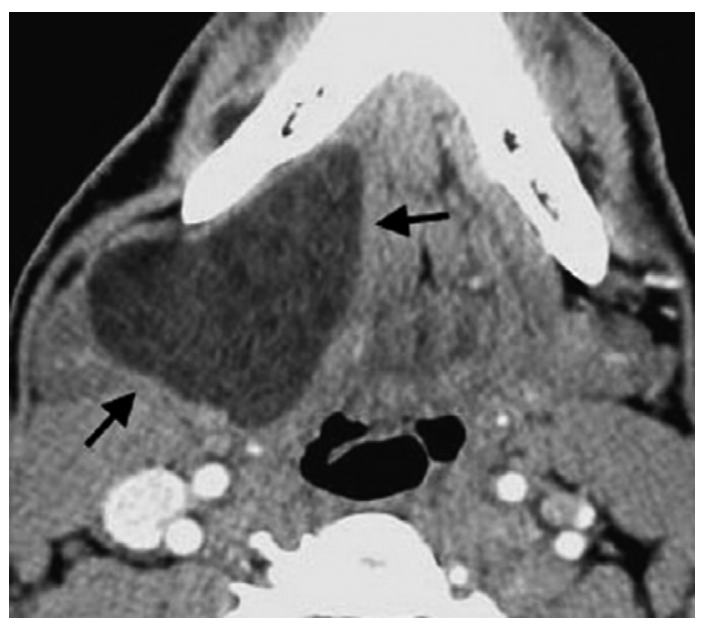

Fig. 6. "Sack of marbles" appearance of a dermoid cyst, as seen on a computed tomography scan (arrows). (From Friedman ER, John SD. Imaging of pediatric neck masses. Radiol Clin North Am 2011. https://doi.org/10.1016/j.rcl.2011.05.005; 49(4):617-32; with permission.) 


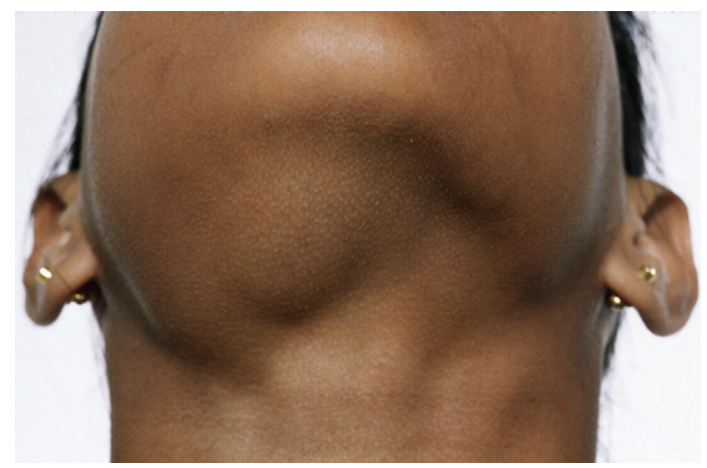

Fig. 7. Plunging ranula. (From Mahadevan M, Vasan N. Management of pediatric plunging ranula. Int J Pediatr Otorhinolaryngol 2006;70(6):1049-54; with permission.)

is paramount to ensuring complete excision and avoiding injury to the surrounding structures.

\section{Midline cervical clefts}

Midline congenital anomalies are congenital neck lesions that result from impaired fusion of branchial arches in the anterior neck are called midline cervical clefts. ${ }^{7}$ They can extend from mandible to manubrium, presenting as a linear vertical area of thin and erythematous mucosa at birth. ${ }^{7}$ There is often a projection from the upper portion of the lesion and a sinus or fistula inferiorly. ${ }^{7}$ Sometimes, there is a fibrous band beneath the mucosal defect, as well ${ }^{7}$ (Fig. 8). If not treated early, the midline cord begins to tether the anterior neck as the infant grows. ${ }^{7}$ Thus, surgical excision has both cosmetic and functional benefits.

\section{LATERAL CONGENITAL NECK MASSES Branchial Cleft Anomalies}

Branchial arches form the embryologic precursors of the ear, as well as the muscles, vasculature, bones, cartilage, and mucosal lining of the face, neck, and pharynx. ${ }^{8}$ Branchial arch anomalies are the second most common head and neck congenital lesions in children and represent approximately $20 \%$ of cervical masses in the pediatric population. ${ }^{8}$ Second branchial arch anomalies are the most common and account for approximately $95 \%$ of cases. ${ }^{8}$

Branchial cleft anomalies arise from incomplete obliteration of any branchial tract, resulting in a cyst, a sinus, or a fistula. Branchial cleft cysts are fluid filled, and the material expressed from them may resemble brown motor oil. They are commonly wellcircumscribed, painless, and mobile. Branchial cleft cysts have no external opening. Branchial cleft sinus tracts occur from incomplete closure of clefts and pouches, yet have single body surface communication, either to pharynx or skin. ${ }^{1}$ Branchial cleft fistulas form similarly, but communicate with 2 body surfaces. ${ }^{1}$ Cysts more commonly present in older children, whereas fistulas are more often recognized in infancy or younger children. ${ }^{8}$

\section{First Branchial Cleft Anomalies}

First branchial cleft cysts are the most cephalad of the anomalies. They are rare $(<1 \%)$, typically originate from the angle of the mandible, extend to the external auditory canal, and are often associated with the facial nerve. ${ }^{1}$ First branchial cleft 


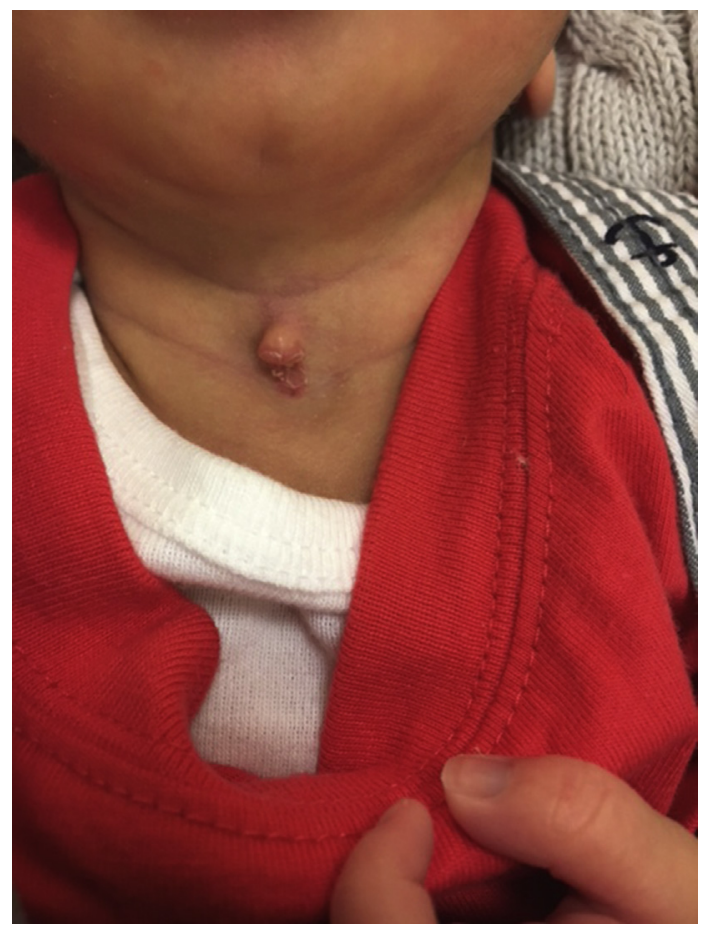

Fig. 8. A 3-month-old infant with a midline cervical cleft.

cysts are further characterized as either Work type I (preauricular, extending to ear canal or middle ear) or Work type II (angle of mandible, extending to concha or ear canal). ${ }^{1}$

\section{Second Branchial Cleft Anomalies}

Second branchial cleft anomalies are the most common (95\%) of the 4 types, with cysts occurring more frequently than sinuses or fistulas. ${ }^{1}$ Unilateral and right-sided presentations are most common. ${ }^{1}$ Second branchial cleft anomalies are located in close proximity to the internal jugular vein. ${ }^{7}$ Fistulas open to the skin along the anterior border of the SCM muscle, dive deep between second and third branchial arch structures to course between the external and internal carotid arteries, lateral to glossopharyngeal and hypoglossal nerves, medial to posterior belly of the digastric and stylohyoid, and ultimately connect to the tonsillar fossa. ${ }^{1}$ Second branchial cleft anomalies can be associated with branchiootorenal syndrome, so auditory and renal screening is important upon diagnosis of this particular congenital neck lesion.

\section{Third and Fourth Branchial Cleft Anomalies}

Like first branchial cleft anomalies, third and fourth branchial cleft cysts are rarely encountered. They are typically located lower in the neck than second branchial cleft cysts, along the inferior-most one-third of the anterior border of the SCM muscle. Sinus tracts, if present, ascend along the carotid sheath posteriorly toward the internal carotid artery, under the glossopharyngeal nerve, and over the vagus and hypoglossal nerves, to open into the piriform sinus or thyrohyoid membrane. 
Third and fourth branchial cleft anomalies are distinguished anatomically by their relationship to the superior laryngeal nerve with third pharyngeal cleft anomalies above the nerve, and fourth pharyngeal cleft anomalies below. ${ }^{8}$ Most third branchial cleft cysts present in the posterior cervical space (posterior to the SCM muscle) as painless, fluctuant masses that may enlarge and become tender if infected. ${ }^{8}$ An infected third branchial cleft cyst should be considered when a pediatric patient presents with an abscess in the posterior triangle of the neck. ${ }^{8}$

A fourth branchial cleft fistula or sinus tract arises from the pyriform sinus apex and descends inferiorly into the mediastinum along the path of the tracheoesophageal groove. ${ }^{8}$ These branchial cleft anomalies are commonly left sided and most often present as a sinus tract coursing from the apex of the pyriform fossa to the upper aspect of the left thyroid lobe. ${ }^{8}$

\section{Management of Branchial Cleft Anomalies}

Surgical excision is curative and a commonly chosen option for treatment of branchial cleft anomalies, particularly if there is recurrent infection. ${ }^{8}$

\section{Imaging of Branchial Cleft Anomalies}

Usually, branchial cleft anomalies are imaged with MRI with contrast (Fig. 9). If an overt pit or fistula is evident on examination and amenable to cannulation, then CT neck with fistulogram and 3-dimensional reformatting is preferred (Fig. 10).

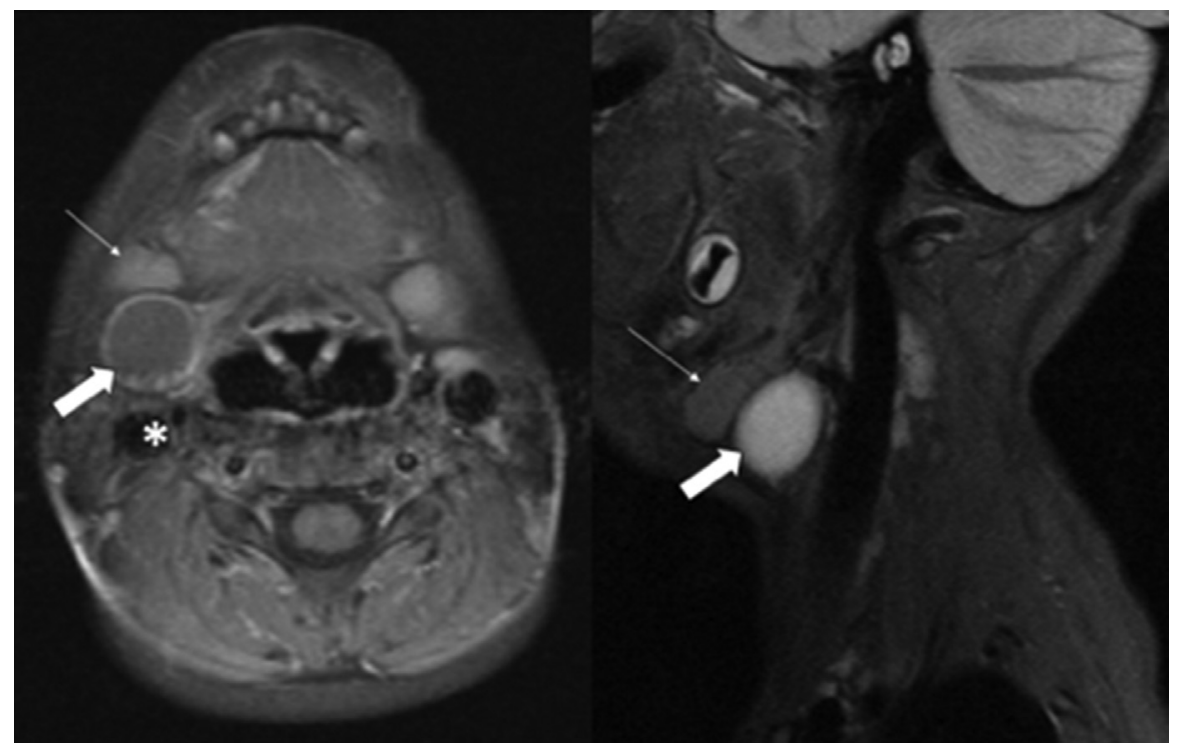

Fig. 9. A 2-year-old child with axial fat-suppressed T1-weighted postcontrast and sagittal short tau inversion recovery images demonstrating a rounded and well-defined T1-weighted isointense, T2-weighted hyperintense lesion with thin peripheral enhancement (thick white arrows). This lesion is demonstrated posterior to the right submandibular gland (thin white arrow) and anterior to the sternocleidomastoid muscle and carotid sheath (asterisk). This diagnosis was confirmed on surgical excision to represent a second branchial cleft cyst. (From Adams A, Mankad K, Offiah C, et al. Branchial cleft anomalies: a pictorial review of embryological development and spectrum of imaging findings. Insights Imaging 2016;7(1):69-76; with permission.) 


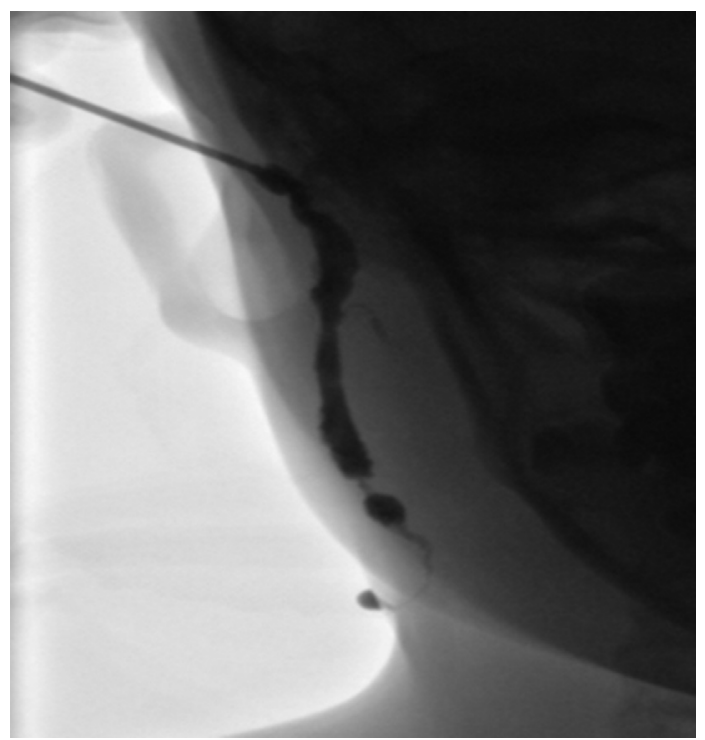

Fig. 10. A sinogram performed on a child before surgical excision of a presumed first branchial cleft fistula. The opening within the right external auditory canal was cannulated and water-soluble contrast media was injected, with sinography confirming the presence of a fistulous tract. During the procedure, contrast media was noted to pass via the tract to an external cutaneous opening in the right submandibular region. (From Adams A, Mankad $\mathrm{K}$, Offiah C, et al. Branchial cleft anomalies: a pictorial review of embryological development and spectrum of imaging findings. Insights Imaging 2016;7(1):69-76; with permission.)

\section{Torticollis}

Torticollis, also known as fibromatosis colli, is an in utero contraction of the SCM muscle with fibrous infiltrate, which may present as a neck mass in infancy. The neonate's head is typically tilted laterally toward the ipsilateral affected muscle and the chin is rotated contralateral to the contracture (Fig. 11). CT scanning or ultrasound examination shows an isodense oval mass in the SCM muscle. ${ }^{1}$ Torticollis can lead to positional plagiocephaly. Physical therapy can help torticollis tremendously. Parents should also be advised to place stimuli on the child's affected side to encourage greater neck range of motion. Imaging and surgical are warranted only if the condition persists beyond the first year of life.

\section{Thymic Cysts}

Cervical thymic cysts, like their thyroglossal duct corollary, arise from persistence of the embryologic thymopharyngeal duct, which can occur adjacent to the carotid sheath anywhere from the hyoid bone to the anterior mediastinum. ${ }^{4}$ The most common age of presentation of a cervical thymic cyst is 2 to 15 years, with a slight male predilection. ${ }^{4}$ Cervical thymic cysts may have a similar appearance to third and fourth branchial cleft cysts, being differentiated only by the presence of thymic tissue upon excision. ${ }^{4}$ The cysts usually present as a painless, unilocular cystic mass, extending inferiorly within the neck, paralleling the SCM muscle. ${ }^{4}$ They can be found anywhere from the angle of the mandible to the thoracic inlet or mediastinum, more typically on the left side. ${ }^{9}$

Subcategories of cervical thymic lesions include ectopic or undescended thymus (typically not cystic), accessory thymus, cervical thymic cysts, thymopharyngeal 


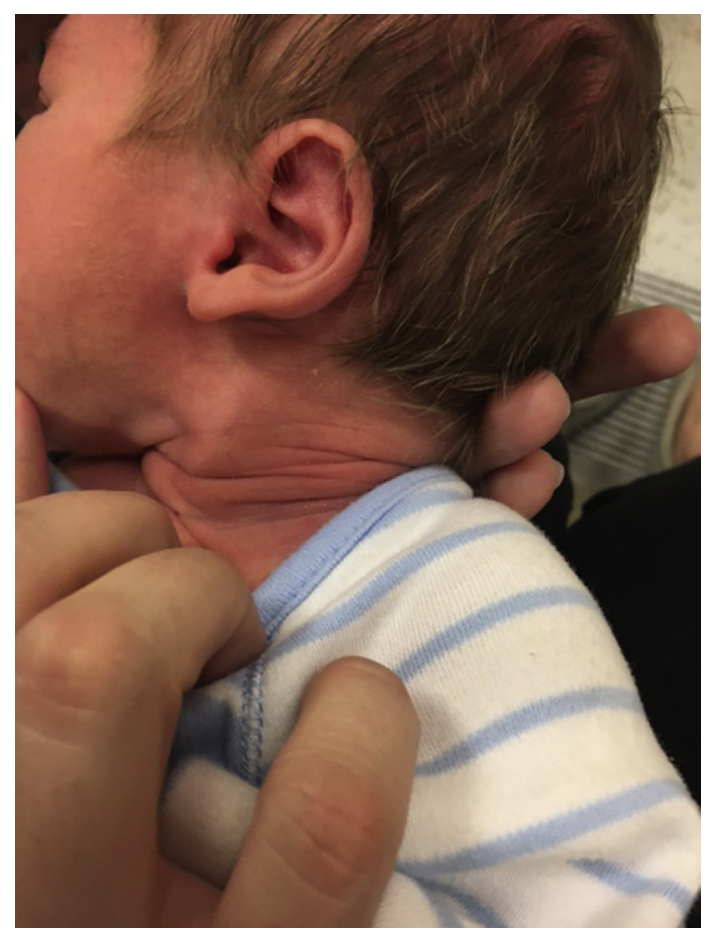

Fig. 11. An 8-week-old infant with a left midsternocleidomastoid mass consistent with congenital torticollis.

duct cyst, and cervical extension of mediastinal thymus (with midline thymus at thoracic inlet). ${ }^{10} \mathrm{CT}$ scanning or MRI with contrast are standard imaging for diagnosis; the specific study varies by patient and institution. Surgical excision of thymic cysts is typically indicated.

\section{Laryngocele}

A laryngocele is formed by a congenital herniation of the saccule of the larynx, and more commonly presents in adulthood rather than the pediatric population. ${ }^{1}$ This herniation can be limited to the anatomic boundaries of the larynx (internal laryngocele), or extend through the thyrohyoid membrane (external or mixed laryngocele). ${ }^{1}$ When a laryngocele extends beyond the larynx, it often presents as an anterior neck cyst that episodically fills with air. ${ }^{1}$ The etiology is suspected to be from a congenital enlargement of the laryngeal saccule, followed by a period of prolonged increased laryngeal pressure (from straining or crying) with a partial obstruction of the neck of the saccule, trapping air within the herniated tissue ${ }^{1}$ (Fig. 12). An internal laryngocele presents more commonly in infancy as stridor, respiratory distress, feeding problems, and/or chronic cough. In the pediatric population, external laryngocele most commonly presents during the teen years in conjunction with playing a musical instrument that requires increased laryngeal pressure, such as the trumpet. External laryngoceles may form a visible or palpable mass in the neck. ${ }^{11}$ Evaluation, particularly with presenting symptoms of airway compromise, may require fiberoptic or direct laryngoscopy. A CT scan with contrast is the most accurate imaging modality for defining spatial relationships between a laryngocele and laryngeal structures, as well as 

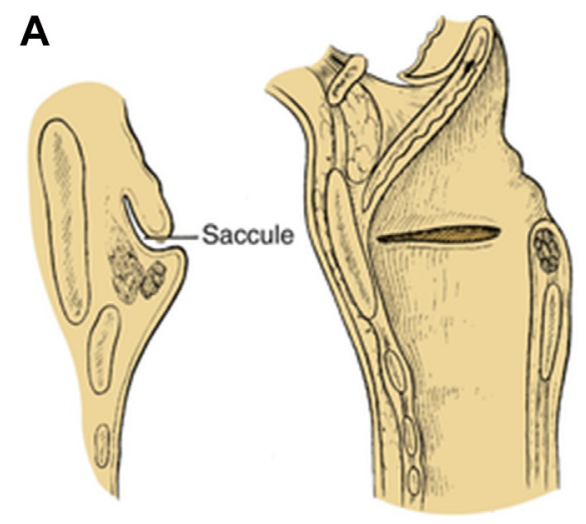

B

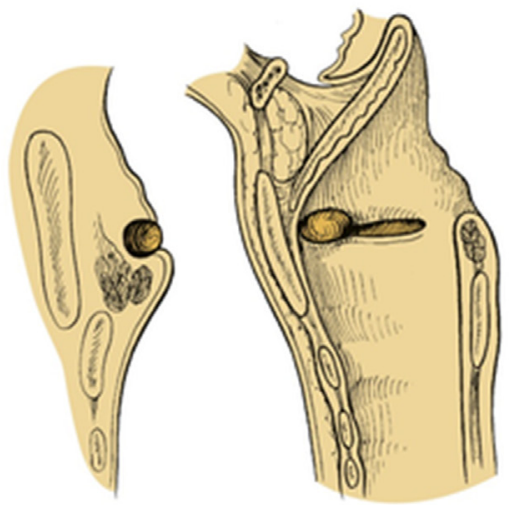

C
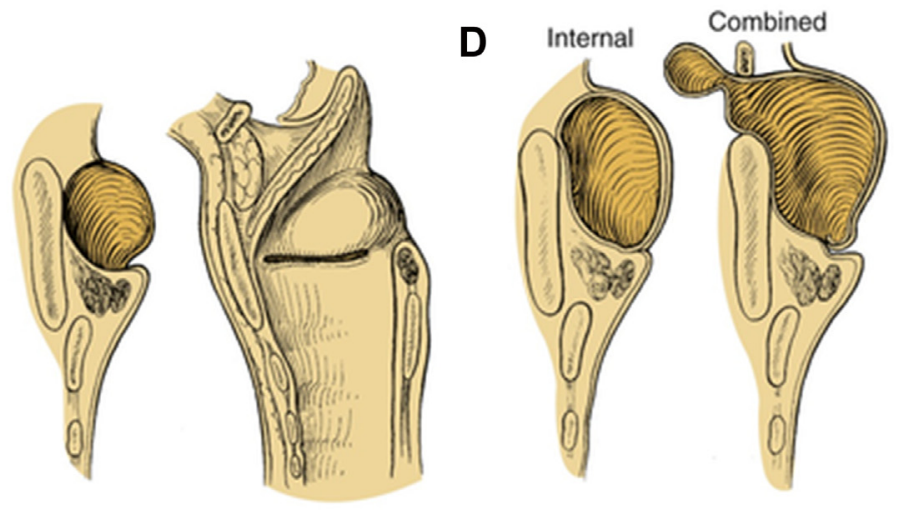

Fig. 12. The classification scheme for a saccular cyst or laryngocele. $(A)$ Normal anatomy. $(B)$ Anterior saccular cyst. $(C)$ Lateral saccular cyst. $(D)$ Laryngocele types. Benign vocal fold mucosal disorders. (From Richardson M, Flint P, Haughey B, et al. Diagnostic imaging of the pharynx and esophagus. Cummings otolaryngology head \& neck surgery. Philadelphia: Elsevier; 2010. https://doi.org/10.1016/B978-0-323-05283-2.00063-X. Fig 62-27; with permission.)

extralaryngeal soft tissues, in differentiating a laryngocele from other cystic formations, and in identifying the coexistence of any laryngeal malignancy ${ }^{11}$ (Fig. 13). Management, based on severity of symptoms, may include respiratory support with the possible need for intubation and/or tracheostomy, observation, cyst decompression via needle aspiration, endoscopic resection, or resection through an external approach. ${ }^{11}$

\section{CENTRAL AND/OR LATERAL CONGENITAL NECK MASSES Vascular Anomalies}

Vascular lesions of the pediatric neck are typically characterized as either hemangiomas or vascular malformations. ${ }^{12}$

\section{Hemangiomas}

Congenital hemangiomas are differentiated from infantile hemangiomas in that congenital hemangiomas are fully formed at birth and do not go through a regression 


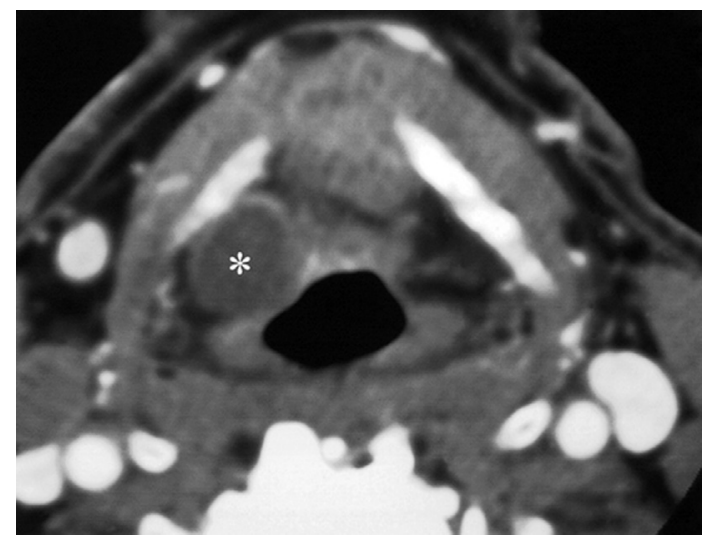

Fig. 13. Intrinsic laryngocele. Enhanced computed tomography of the larynx reveals a fluidfilled mass (asterisk) in the paraglottic fat. Laryngoceles may be filled with air or fluid. (From Richardson $M$, Flint $P$, Haughey $B$, et al. Diagnostic imaging of the pharynx and esophagus. Cummings otolaryngology head \& neck surgery. Philadelphia: Elsevier; 2010. p. 1393-420. Fig 102-18.)

phase. ${ }^{1}$ Infantile hemangiomas present soon after birth and undergo proliferation, then involution stages. ${ }^{1}$

\section{Infantile hemangiomas}

Infantile hemangiomas may present at birth as flat, violaceous lesions that may be mistaken for a bruise. They become more prominent with crying or straining. They expand by early proliferation and begin to spontaneously involute around 12 months of age, typically never to regrow. ${ }^{1}$ The proliferation stage usually starts at 2 to 4 weeks of age, is most rapid in the first 4 to 6 months, and continues through about 1 year of age. ${ }^{1}$ Growth of infantile hemangiomas may continue in some cases after a year of age, particularly when involving the airway, when in a beard distribution, or when treated with high-dose steroids. ${ }^{1}$ The involution phase starts at about 1 year of age and rate can be extremely variable, ranging from a quick resolution to slow improvement over 10 years. ${ }^{1}$

\section{Congenital hemangiomas}

Congenital hemangiomas are fully formed at birth and may be identified on prenatal ultrasound imaging. These lesions are raised, and usually pink to blue color. There are 2 subtypes of congenital hemangiomas: rapidly involuting congenital hemangioma and never involuting congenital hemangioma. ${ }^{1}$ Rapidly involuting congenital hemangioma are hemangiomas that are fully developed at birth and immediately begin to involute. Never involuting congenital hemangioma are also fully developed at birth, but maintain their shape and size, and never involute. ${ }^{13}$ Involution of rapidly involuting congenital hemangiomas takes place at an estimated rate of $10 \%$ per year, so that approximately $50 \%$ have involuted by 5 years of age, $70 \%$ by 7 years, and $90 \%$ by 9 years. ${ }^{13}$ An association has been shown between cervicofacial hemangiomas in a beard distribution and subglottic and upper airway hemangiomas; patients with 2 or more hemangiomas along the jawline should be observed for stridor or a crouplike cough. Laryngoscopy is critical at the first sign of stridor or croup in these patients. ${ }^{14}$

PHACES syndrome should be considered in patients with 2 or more of the following for which it is named: posterior fossa abnormalities, hemangioma, arterial abnormalities, cardiac abnormalities, eye abnormalities, and/or sternal cleft or defect. ${ }^{1}$ PHACES 
has been reported in patients with segmental facial hemangiomas as well as in those with isolated, focal hemangiomas ${ }^{1}$ (Fig. 14). The workup consists of MRI and MR angiography of neck and brain, echocardiogram, and an ophthalmologic assessment. ${ }^{1}$

Diffuse neonatal hemangiomatosis is a rare condition characterized by the presence of numerous cutaneous and visceral hemangiomas that manifest at birth or within the neonatal period. The cutaneous lesions are generalized, vary from 0.5 to $1.5 \mathrm{~cm}$ in diameter, and range from 50 to 500 in number. Visceral lesions are most commonly found in the liver, central nervous system, intestine, and lungs. Skeletal involvement has also been reported. Approximately $60 \%$ of infants with diffuse neonatal hemangiomatosis die during the first few months of life owing to high-output cardiac failure, hemorrhage, or central nervous system involvement. ${ }^{1}$ Steroid therapy is thought to accelerate involution of diffuse neonatal hemangiomatosis lesions. ${ }^{15}$

Management of hemangiomas depends on extent and location of involvement; initially, watchful waiting is often reasonable, because the majority of these lesions resolve spontaneously and the disease process is benign. ${ }^{1}$ However, for hemangiomas that are deforming, impair function, or are ulcerated, propranolol (Inderal) has become the first line of therapy with vasoconstriction as the therapeutic objective. ${ }^{1}$ This agent should be used in caution in patients with PHACES syndrome. ${ }^{1}$ Additional treatment modalities for congenital hemangiomas include topical timolol gel, intralesional steroid injections, chemotherapeutic agents, and laser treatments. Hemangiomas involving the neck and face, although benign, can lead to parental stress, fear, and grief. This factor should be considered when caring for patients with facial hemangioma and their families.

\section{Vascular Malformations}

Vascular malformations may or may not be present at birth. ${ }^{1}$ These lesions do not involute, but rather will grow with the patient, and so surgical excision is typically required. $^{12}$ Several different types of vascular malformations are recognized:

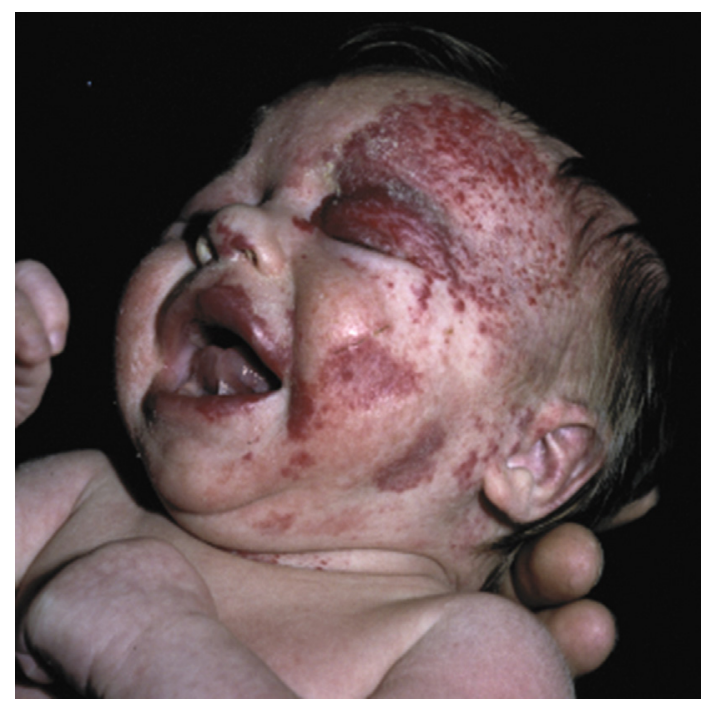

Fig. 14. Hemangioma associated with PHACES (posterior fossa malformations-hemangiomas-arterial anomalies-cardiac defects-eye abnormalities-sternal cleft and supraumbilical raphe) syndrome. (From Conlon JD, Drolet BA. Skin lesions in the neonate. Pediatr Clin North Am 2004;51(4):863-88, vii-viii; with permission.) 
arteriovenous malformations, venous malformations, capillary malformations, and lymphatic malformations. ${ }^{1}$ For vascular anomalies, diagnostic ultrasound can be helpful by determining intraluminal flow and flow voids, but neck MRI with gadolinium and fat suppression, sometimes with MR angiography, is the gold standard. ${ }^{1}$ Arteriovenous malformation lesions typically show an infiltrative mass, bright on T1-weighted imaging, along with tissue infiltration and destruction. ${ }^{1}$ Both venous and lymphatic malformations exhibit slow flow and enhance on T2-weighted imaging. ${ }^{1}$ Ultrasound examination of lymphatic malformations will show a complex, multilocular cystic mass; MRI shows low to intermediate intensity on T1-weighted images, and hyperintensity on T2-weighted images. ${ }^{1}$

\section{Arteriovenous malformations}

Arteriovenous malformations are complex masses of arteries and veins associated with rapid blood flow and a high risk of recurrence after treatment. ${ }^{12}$ An associated bruit may be present on auscultation. These classically have a superficial blue to bluish-red color and will undergo aggressive growth, resulting in tissue destruction ${ }^{1}$ (Fig. 15). Embolization is the treatment of choice, for the purpose of limiting growth and control of acute bleeding. ${ }^{1}$

\section{Capillary malformations}

Capillary malformations, commonly known as port wine stains, cause light pink to dark red discoloration of the skin. ${ }^{12}$ These lesions are typically noted at birth, but differ from

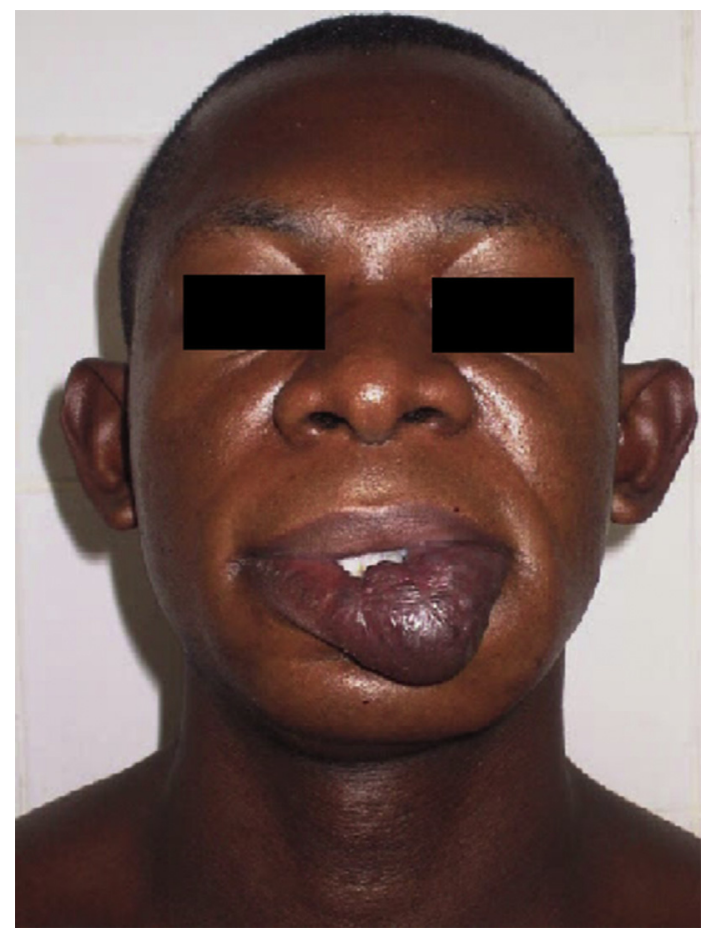

Fig. 15. Arteriovenous malformation of the lower lip. A tobacco-pouch suture technique for the treatment of vascular lesions of the lip in Enugu, Nigeria. (From Oji C, Chukwuneke F, Mgbor N. Tobacco-pouch suture technique for the treatment of vascular lesions of the lip in Enugu, Nigeria. Br J Oral Maxillofac Surg 2006;44(3):245-7; with permission.) 
hemangiomas in that they do not undergo a proliferative phase. ${ }^{1}$ The mainstay of management is serial pulsed dye laser treatment or surgery to treat soft tissue hypertrophy. ${ }^{1}$ Facial venous malformations of the forehead, scalp, and eye in V1 and V2 distribution should raise index of suspicion for Sturge-Weber syndrome, also known as encephalotrigeminal angiomatosis, with ocular and central nervous system venous malformations and related impairments.

\section{Venous malformations}

Venous malformations are usually deep blue to purple, collapsing with pressure, and swelling with exertion. ${ }^{1}$ They can be exacerbated by pregnancy, puberty, menopause, or trauma. ${ }^{1}$ Treatment options include laser, sclerotherapy, or surgery. ${ }^{1}$

\section{Lymphatic malformation}

Lymphatic malformations (also known as cystic hygroma or lymphangioma) are most commonly found in the posterior cervical triangle of the neck (Fig. 16) or submandibular space. ${ }^{1}$ These are typically present at birth, at which point approximately $50 \%$ are diagnosed, with $90 \%$ are identified by the age of 2 years. ${ }^{1}$ On physical examination, they have a rubbery, doughlike consistency and do not have well-circumscribed borders, because they do not respect fascial planes. Their violaceous appearance and translumination property distinguish them from other lesions. ${ }^{1}$ Lymphatic malformations can be microcystic (capillary lymphangiomas), macrocytic (cavernous

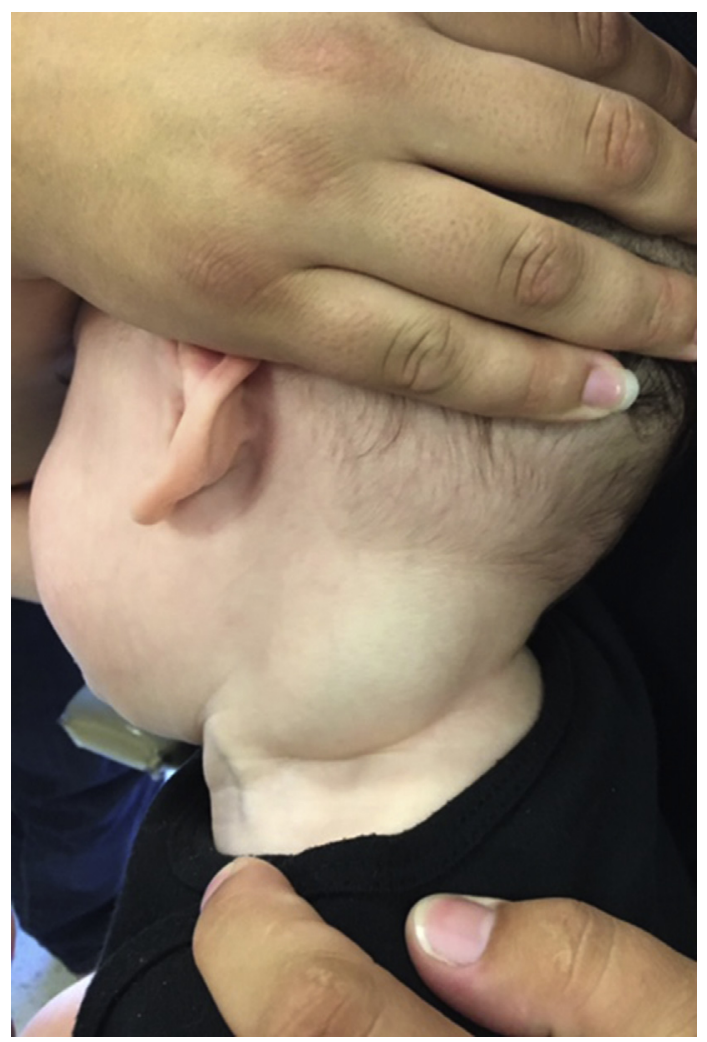

Fig. 16. A 9-month-old infant with a lymphatic malformation of level $\vee$ of left neck. 
lymphangiomas), or mixed. Spontaneous regression occurs in up to $15 \%$ of cases for macrocystic lesions. ${ }^{1}$ Smaller, asymptomatic macrocystic lymphatic malformations can be observed for up to 24 months. ${ }^{1}$ Treatment options for macrocystic lymphangiomas include sclerotherapy with substances such as OK-432 (Picibanil) and/or surgical excision. ${ }^{1}$ Microcystic lymphatic malformations are generally not amenable to sclerotherapy and may require serial excision.

\section{Fetal Cervical Lesions}

Cervical lesions presenting in the fetal period are often giant in size and may have various etiologies, with cervical lymphangiomas and cervical teratomas being the two most common.

\section{Fetal lymphatic malformations}

Lymphatic malformations (described in greater detail elsewhere in this article) may be identified prenatally on fetal ultrasound examination as multiloculated cystic masses that do not respect fascial planes and are not well-circumscribed. ${ }^{1}$ Fetal lymphatic malformations that present earlier, during the second trimester, commonly arise from the posterior triangle and have a high rate of association with chromosomal abnormalities (eg, Turner syndrome). ${ }^{1}$ With later presentation of fetal lymphangioma (during the third trimester or postnatally), there is less likelihood of an associated chromosomal abnormality, and the location is more commonly within the anterior triangle. $^{1}$

\section{Fetal cervical teratomas}

Fetal cervical teratomas of the head and neck are uncommon, comprising less than $5 \%$ of all teratomas. ${ }^{1}$ They may be found in the second trimester on fetal ultrasound examination and typically present as a rapidly enlarging lateral or midline neck mass, which frequently causes airway obstruction. ${ }^{1}$ Teratomas have more defined borders than lymphangiomas and a more heterogenous appearance. ${ }^{1}$ They are associated with maternal polyhydramnios in $30 \%$ of cases. ${ }^{1}$

Management of these very large fetal neck masses involves a multidisciplinary, carefully planned surgical birth via ex utero intrapartum treatment (EXIT procedure) to deliver the head and neck, secure the neonate's airway with tracheostomy, then complete the delivery. ${ }^{1}$ Complete excision of the cervical teratoma or lymphangioma can then be performed ${ }^{1}$ (Fig. 17).

\section{INFECTIOUS AND INFLAMMATORY NECK MASSES}

Acute inflammatory or infections neck masses, by definition, are those present for less than 2 weeks, and chronic masses have been present for 2 weeks or more. ${ }^{1}$

\section{Acute Infectious and Inflammatory Neck Masses}

\section{Cervical lymphadenitis}

Two of the most common viral causes of pediatric cervical lymphadenopathy are Epstein-Barr virus and cytomegalovirus. Additional culprits include influenza, parainfluenza, rhinovirus, adenovirus, and coronavirus. Pediatric viral exanthems such as mumps, measles, and coxsackie viruses can also cause cervical lymphadenopathy. Bacterial etiologies include Streptococcus pyogenes and Staphylococcus aureus, although methicillin-resistant $S$ aureus is increasing. Sources of infection resulting in cervical lymphadenopathy include otitis media, adenoiditis, tonsillitis, and skin and scalp lesions. Infectious and inflammatory lymphadenopathy is usually tender. There are often associated constitutional symptoms, including malaise and fever. If 


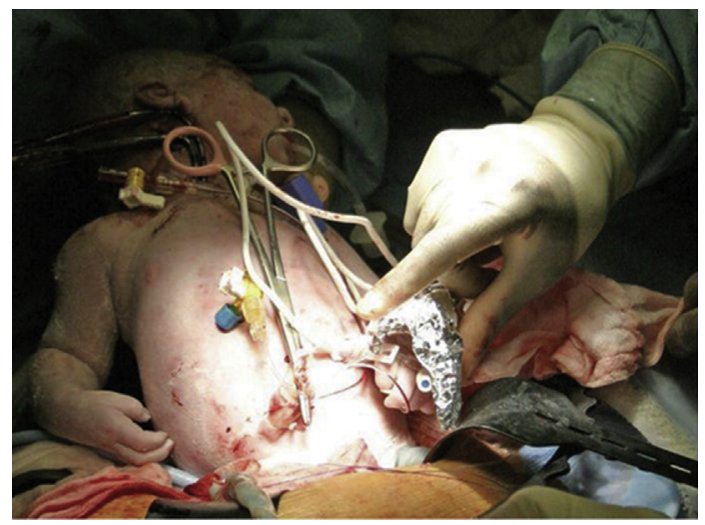

Fig. 17. Ex utero intrapartum treatment procedure. (From Marwan A, Crombleholme T. The EXIT procedure: principles, pitfalls, and progress. Semin Pediatr Surg 2006;15(2):107-15; with permission.)

resolution of lymphadenopathy occurs with use of antibiotic therapy, no further laboratory tests or imaging studies are typically required. When lymphadenopathy is unresponsive to oral antibiotics, observation, incision and drainage, pertinent laboratory tests, and/or imaging studies may be warranted.

\section{Chronic Infectious and Inflammatory Neck Masses}

\section{Atypical mycobacterial infection}

Atypical mycobacterial infection presents in the pediatric neck as a painless, fluctuant lymph node with a violaceous appearance of the overlying skin, and eventual breakdown of skin with necrosis ${ }^{1}$ (Fig. 18). This organism is found in dirt or unpasteurized milk products. ${ }^{1}$ Infected individuals typically have a positive PPD test result. ${ }^{1}$ Treatment includes antibiotic therapy, typically a macrolide, in conjunction with an antimycobacterial agent such as rifampin (Rafidan) for 12 weeks at minimum. Surgical excision is ultimately necessary and pathology classically shows necrotizing granulomatous inflammation, staining positive for acid-fast bacilli.

\section{Toxoplasmosis}

Toxoplasmosis is a disease caused by a very common parasite, Toxoplasma gondii. ${ }^{1}$ Infection with this parasite can be acquired by changing cat litter boxes, consuming undercooked meats (from an infected animal), through blood transfusion, or via placental transmission. ${ }^{1}$ Infected children may be asymptomatic or can present with flulike symptoms and cervical lymphadenitis. A diagnosis of toxoplasmosis is made via antibody testing, with elevated levels of immunoglobulin $M$ (earlier disease) and immunoglobulin G (later disease). ${ }^{1}$ The condition is typically self-limited unless the patient is immunocompromised. ${ }^{1}$

\section{Bartonella}

Cat scratch disease can develop over weeks to months in children who have had exposure to cats, with or without a recalled history of a cat scratch. ${ }^{1}$ Serologic testing for $B$ henselae is positive in infected individuals, who present with a painless and fluctuant cervical mass. The condition is self-limited, although treatment with antibiotics may be recommended, particularly for immunocompromised children. ${ }^{1}$ 


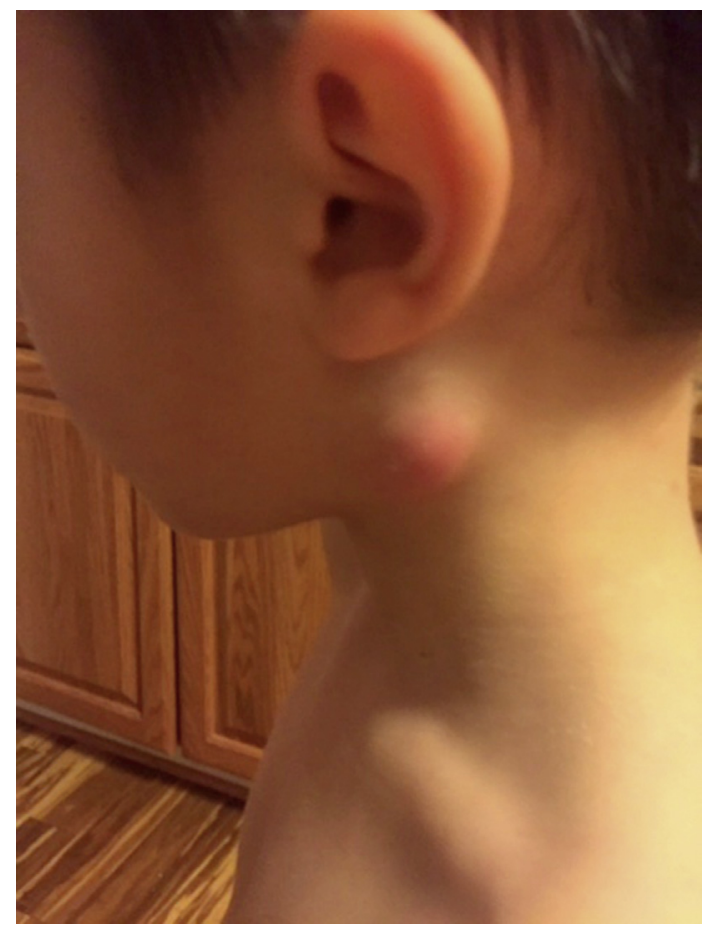

Fig. 18. A 6-year-old child with an atypical mycobacteria infection.

\section{Human immunodeficiency virus}

HIV infection commonly causes generalized lymphadenopathy, often involving the cervical and occipital nodes. ${ }^{1}$ Identification obtained via HIV serology and treatment is per infectious disease protocol.

\section{Tick-Borne Illnesses}

\section{Lyme disease}

Lyme disease, the most common vector-borne infection in the United States, is caused by the spirochete Borrelia burgdorferi. ${ }^{16}$ The majority of cases occur in the Northeastern coastal regions, the upper Midwest, and northern California. ${ }^{16}$ Early localized disease is characterized by erythema migrans, classically an erythematous and enlarging "bulls eye" rash. ${ }^{16}$ Mild systemic complaints, such as fever, fatigue, myalgias, arthralgias, headache, and cervical lymphadenopathy, may accompany erythema migrans. ${ }^{16}$ Positive Lyme antibody screening tests should be confirmed by Western blot test. ${ }^{16}$ Doxycycline (Vibramycin) is recommended for treatment of early localized and early disseminated disease, whereas ceftriaxone (Rocephin) is preferred for Lyme meningitis and late disseminated disease. ${ }^{16}$

\section{Ehrlichiosis and Rocky Mountain Spotted Fever}

Other tick-borne illnesses may present similarly with symptoms including, but not limited to, lymphadenopathy, fatigue, fever, rash, and myalgias. ${ }^{16}$ Ehrlichia chaffeensis is the agent responsible for human monocytic ehrlichiosis. Most reported cases of human monocytic ehrlichiosis infection occur in the South and Southeast, and Amblyomma americanum and Dermacentor variabilis are the 
principal tick vectors. ${ }^{16}$ Rickettsia rickettsia is the etiologic agent of Rocky Mountain Spotted Fever, which found in $D$ variabilis (the dog tick) in Southeastern and Western states, and in $D$ andersoni (the wood tick) in Rocky Mountain states. ${ }^{16}$ Testing for both conditions involves enzyme-linked immunosorbent assay or polymerase chain reaction testing, and treatment is typically with oral or intravenous tetracycline. $^{16}$

\section{NEOPLASTIC NECK MASSES \\ Benign Neck Neoplasms}

\section{Pilomatrixoma}

Hair follicle matrix cells may form pilomatrixomas, most typically in the suboccipital region or the posterior triangle ${ }^{17}$ (Fig. 19). These lesions present as superficial, rockhard, mobile neck masses and may have a bluish hue. They are slow growing, with a female preponderance. ${ }^{1}$ Pilomatrixoma should be differentiated from epidermal and dermoid cysts; the latter two are less dense, deeper, and have normal overlying skin. ${ }^{17}$ Pilomatrixomas slide freely over the underlying tissues; often, a "tent sign" can be elicited by stretching the skin over the tumor to feel the irregular surface of the mass. ${ }^{17}$ Diagnosis can be made clinically and surgical excision is curative.

\section{Lipomas}

Lipomas are benign lesions and are rare in children. They consist of adipose tissue, and are painless, soft, and mobile on examination. These lesions can be excised surgically for functional or cosmetic purposes, if desired, because they may enlarge gradually.

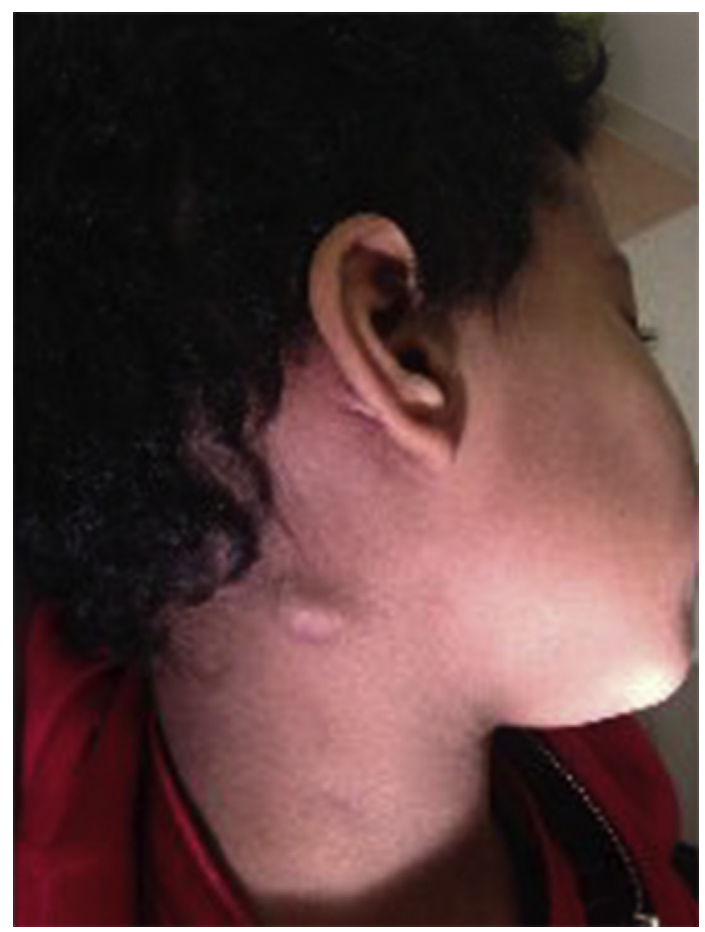

Fig. 19. A 5-year-old child with a pilomatrixoma. 


\section{Neural tumors}

Benign neural tumors commonly involve cranial nerves and can present as isolated lesions or as part of a comprehensive syndrome. Neurofibromas (schwannomas), for example, may occur in autosomal dominant disorders. ${ }^{1}$ Neurofibromatosis type 1 or von Recklinghausen disease is characterized by extensive nerve sheath tumor development and café-au-lait spots. ${ }^{1}$ Children with neurofibromas of the head and neck should undergo imaging and genetic testing to rule out neurofibromatosis. ${ }^{1}$

\section{MALIGNANT NECK NEOPLASMS AND METASTATIC LYMPHADENOPATHY}

Malignant neck masses in children are uncommon. Several of the leading causes of metastatic cervical nodes in children are discussed below. Imaging modalities for the evaluation of acute lymphoblastic leukemia potentially malignant neck neoplasms involve ultrasound examination or contrast CT scanning with or without MRI. Treatment of pediatric malignant neck lesions typically consists of surgical excision and chemoradiation.

\section{Nasopharyngeal Carcinoma}

Nasopharyngeal carcinoma is one of the most common nasopharyngeal tumors in young children. ${ }^{18}$ It is distinguished from the adult form of the disease by its association with Epstein-Barr virus infection, undifferentiated histology, and high incidence of advanced locoregional compromise. ${ }^{18}$ Painless, posterior cervical, metastatic nodes are typically seen with nasopharyngeal carcinoma. ${ }^{18}$ It rarely occurs in children under 14 years of age. ${ }^{18}$ Although enormous differences exist among races and geographic groups, nasopharyngeal carcinoma makes up $1 \%$ to $5 \%$ of all pediatric cancers and $20 \%$ to $50 \%$ of all primary malignant nasopharyngeal tumors in children. ${ }^{18}$ There is a higher incidence in teenage males, those of Asian descent, and among children of Western industrial nations. ${ }^{18}$ Patients may present with epistaxis and nasal obstructive symptomatology warranting evaluation with nasal endoscopy. ${ }^{18}$ In children and adolescents, neoadjuvant chemotherapy and subsequent radiotherapy are preferred, but the best results are obtained with interferon- $\beta$, which is expensive and is not widely available in low-income countries. ${ }^{18}$

\section{Lymphoma}

Lymphoma is the most common malignant pediatric neck mass, reported to have a male predominance. ${ }^{1}$ Non-Hodgkin lymphoma presents more commonly in children less than 10 years of age. Hodgkin lymphoma is more common in older children. ${ }^{1}$

Hodgkin lymphoma is a neoplastic disease of the lymphatic cell line and is distinguished histologically from non-Hodgkin lymphoma by its characteristic ReedSternberg cells. ${ }^{19}$ Swollen, rubbery, nontender lymph nodes are frequently the most noticeable initial sign. ${ }^{19}$ Histologic examination reveals Hodgkin and multinucleated Reed-Sternberg cells and multicolor lymphocytic reactions. ${ }^{19}$ Anamnesis and clinical examination provide diagnostic indicators; staging is performed as in non-Hodgkin lymphoma with imaging, bone marrow aspiration, and histologic evidence of biopsied tissue. ${ }^{19}$ Chemotherapy and, if applicable, radiotherapy are used as treatment options, according to the stage of the disease. ${ }^{19}$

Non-Hodgkin lymphomas are a heterogeneous group of neoplastic disorders of the lymphatic cell, which affects B-cell and T-cell lymphocytes. ${ }^{19}$ The etiology is considered to be hereditary or acquired genetic defects leading to a mismatch of proliferation and apoptosis of cells in the lymphatic system. ${ }^{19}$ Lymph node swelling and general symptoms such as fever, weight loss, and night sweats are the 
predominant manifestations. ${ }^{19}$ Patients additionally often exhibit fatigue, weakness, and changes in blood count. ${ }^{19}$ The swollen lymph nodes are usually firm and nontender, and may be unilateral or bilateral. ${ }^{19}$ Biopsy for histologic evaluation with special immune staining and bone marrow aspiration are necessary for diagnosis. Imaging is useful in staging the disease, including chest radiographs, CT scans, and ultrasound imaging of the neck, thorax, and abdomen. ${ }^{19}$ Treatment is based on the stage and entity and may include chemotherapy, radiation, and/or antibody therapy. ${ }^{19}$

\section{Neuroblastoma}

Neuroblastoma is the most common head and neck malignancy in children less than 5 years of age, and it is the most common malignancy diagnosed during the first month after birth. ${ }^{1}$ It can be associated with Horner syndrome and cranial neuropathies, as well as airway obstruction or dysphagia, because neuroblastomas arise in the neural crest progenitor cells of the sympathetic nervous system. ${ }^{1}$ Treatment involves surgery and radiation for stage $A$ disease (confinement to the nasal cavity). Stages B, C, or D disease (paranasal sinus involvement, extension to skull base, and distant metastasis including cervical lymph nodes, respectively) warrants surgery, radiation, and possibly postoperative chemotherapy. ${ }^{1}$

\section{Rhabdomyosarcoma}

Rhabdomyosarcoma is a highly malignant tumor of mesenchymal origin. ${ }^{18}$ This neoplasm is the most common pediatric soft tissue sarcoma of the head and neck, and the second most common head and neck cancer. Rhabdomyosarcoma is typically encountered in children less than 10 years of age. ${ }^{1}$ Upon biopsy, characteristic histologic indicators are eosinophilic cells and multinucleate giant cells. ${ }^{18}$ In most cases, chemotherapy and radiation are initiated, and some cases additionally require surgical excision. ${ }^{1}$

\section{Thyroid Malignancy}

Thyroid nodules are infrequent in children. When present, they are more common in older children, and there is a high likelihood of malignancy. ${ }^{1}$ Adenoma, papillary carcinoma (frequently associated with prior radiation therapy), follicular carcinoma, and medullary thyroid carcinoma can be distinguished via histopathological evaluation. ${ }^{1}$ Multiple endocrine neoplasia syndrome type 2 is an autosomal-dominant, inherited condition that involves medullary thyroid carcinoma, pheochromocytoma, parathyroid hyperplasia (multiple endocrine neoplasia syndrome type 2A) and the addition of mucosal neuromas and marfanoid habitus (in multiple endocrine neoplasia syndrome type $2 \mathrm{~B}$ ). ${ }^{1}$ Prophylactic thyroidectomy is the treatment of choice for multiple endocrine neoplasia syndrome type 2 , as well as for patients with a history of head and neck radiation. $^{1}$

\section{Posttransplant Lymphoproliferative Disorder}

After solid organ transplant, patients on immunosuppression may develop pathologic proliferations of lymphoid tissue, called posttransplant lymphoproliferative disorder. ${ }^{1}$ This condition commonly presents with cervical lymphadenopathy and tonsillar hypertrophy, and is more common in children than adults. ${ }^{1}$ Posttransplant lymphoproliferative disorder can range from lymphoid hyperplasia, such as tonsillar hypertrophy, to malignancy. Biopsy or tonsillectomy is confirmatory, and decreasing immunosuppressive medications is often indicated. ${ }^{1}$ 


\section{OTHER}

Additional etiologic considerations are worth mentioning. Castleman's disease is a proliferation of lymphatic cells commonly associated with HIV and human herpesvirus 8,1 and Rosai-Dorfman disease (sinus histiocytosis) is characterized by nonneoplastic proliferation of histiocytes in the sinusoids of lymph nodes and extranodal tissues. ${ }^{20}$ Hematomas, which are localized collections of extravascular blood, may present after trauma or in association with underlying systemic disease. Uncommon infectious considerations include Lemierre syndrome, a septic thrombophlebitis of the internal jugular vein after infection of pharynx, which is associated with fusobacterium. ${ }^{1}$ Lymphadenopathy may result from tularemia, which can be contracted via rabbits or rodents, although ticks can rarely be a vector.

Additional embryologic remnants of the head and neck to consider include auricular hillocks, which consist of ectopic cartilage deposits, found anywhere from the preauricular space, down the lateral neck, and even into the chest. Auricular hillocks are benign and do not require imaging, but are amenable to surgical intervention if they become infected or bothersome. Cervical bronchogenic cysts (foregut duplication cysts) result from anomalous foregut development, and are usually located in the thyroid or paratracheal region, or rarely in the suprasternal or supraclavicular location. ${ }^{21}$

Malignant salivary gland tumors in children primarily present in the parotid gland, with mucoepidermoid carcinoma being the most common. ${ }^{1}$ These infrequently encountered entities are important to consider, but fall of outside of the scope of this article.

\section{SUMMARY}

Most pediatric neck masses encountered in primary care are benign, reactive lymph nodes that originate from common pediatric viral processes. In a pediatric otolaryngology practice, more unusual pathologies are encountered, such as embryologic anomalies, vascular lesions, or neoplasms. Normal lymph nodes are more easily palpable in children with thin necks, and benign-appearing lymph nodes in children are typically not considered for biopsy unless they are $3 \mathrm{~cm}$ or greater in size. Lesions that are larger or that have concerning features will ultimately need imaging and excisional biopsy for histopathologic confirmation of the diagnosis. A sound clinician understanding of anatomic neck spaces and common etiologies of pediatric neck masses can greatly reduce nonessential testing, cost, delay in treatment, and parental angst.

\section{ACKNOWLEDGMENTS}

The author would like to extend immense gratitude to Dr Stephen Early as her supervising physician and the sole pediatric otolaryngologist at The University of Virginia Medical Center. Thank you for hiring me twice, once in Florida, and then again 15 years later to join your practice at UVA in the Otolaryngology Head and Neck Surgery department. It has been a tremendous honor and privilege to have had the opportunity to work alongside you, truly one of the greatest minds and hearts of all time.

\section{REFERENCES}

1. Parikh S. Pediatric otolaryngology head and neck surgery clinical reference guide. San Diego (CA): Plural Publishing; 2014. p. 422-613.

2. Harish K. Neck dissections: radical to conservative. World J Surg Oncol 2005;3:21. 
3. Meuwley J, Lepori D, Theumann N, et al. Multimodality imaging evaluation of the pediatric neck: techniques and spectrum of findings. Radiographics 2005;25(4): 931-48.

4. Mittal MK, Malik A, Sureka B, et al. Cystic masses of neck: a pictorial review. Indian J Radiol Imaging 2012;22(4):334-43.

5. Cahill AM, Nijs ELF. Pediatric vascular malformations: pathophysiology, diagnosis, and the role of interventional radiology. Cardiovasc Intervent Radiol 2011;34:691.

6. Craniofacial Development. Duke medicine embryology learning resources web site. Available at: https://web.duke.edu/anatomy/embryology/craniofacial/craniofacial. html Copyright (c) 2004-2011 Duke University School of Medicine. Accessed February 23, 2017.

7. Farhadi R, Sahebpour AA, Ghasemi M. Congenital midline cervical cleft: can it be treated in newborn? Iran J Pediatr 2012;22(4):547-50.

8. Adams A, Mankad K, Offiah C, et al. Branchial cleft anomalies: a pictorial review of embryological development and spectrum of imaging findings. Insights Imaging 2016;7(1):69-76. Available at: https://www.ncbi.nlm.nih.gov/pmc/ articles/PMC4729717/. Accessed January 11, 2018.

9. Kaufman MR, Smith S, Rothschild MA, et al. Thymopharyngeal duct cyst: an unusual variant of cervical thymic anomalies. Arch Otolaryngol Head Neck Surg 2001;127(11):1357-60.

10. Shenoy V, Kamath MP, Hedge M, et al. Cervical thymic cyst: a rare differential diagnosis in lateral neck swelling. Case Rep Otolaryngol 2013;2013:350502.

11. Jishana J, Poduval JD. External laryngocele: points to remember. J Laryngol Voice 2013;3(2):67-9.

12. About hemangiomas and vascular birthmarks. EVMS Center for Hemangiomas and Vascular Birthmarks Web site. 2017. Available at: http://www.evms.edu/education/ centers_institutes_departments/otolaryngology_ent/divisions/hemangiomas_and_ vascular_birthmarks/. Accessed March 26, 2017.

13. Interdisciplinary Workgroup for Hemangiomas and Vascular Malformations web site. Available at: http://www.meduniwien.ac.at/haemangiom/index.php?page= 1\&lang=en Medical University of Vienna. Accessed May 26, 2017.

14. Orlow SJ, Isakoff MS, Blei F. Increased risk of symptomatic hemangiomas of the airway in association with cutaneous hemangiomas in a "beard" distribution. J Pediatr 1997;131:643-6.

15. Poirier VC, Ablin DS, Frank EH. Diffuse neonatal hemangiomatosis: a case report. AJNR Am J Neuroradiol 1990;11:1097-9. November/December 1990 0195-6108/90/1 106-1097 (C) American Society of Neuroradiology.

16. Bryant KA, Marshall GS. Clinical manifestations of tick-borne infections in children. Clin Diagn Lab Immunol 2000;7(4):523-7.

17. Danielson-Cohen A, Lin SS, Hughes A, et al. Head and neck pilomatrixoma in children. Arch Otolaryngol Head Neck Surg 2001;127(12):1481-3.

18. González-Motta A, González G, Bermudéz Y, et al. Pediatric nasopharyngeal cancer: case report and review of the literature. Cureus 2016;8(2):e497.

19. Lang S, Kansy B. Cervical lymph node diseases in children. GMS Curr Top Otorhinolaryngol Head Neck Surg 2014;13:Doc08.

20. Lima F, Barcelos P, Constancio A, et al. Rosai-Dorfman disease with spontaneous resolution: case report of a child. Rev Bras Hematol Hemoter 2011;33(4):312-4.

21. Kieran SM, Robson CD, Nose V, et al. Foregut duplication cysts in the head and neck presentation, diagnosis, and management. Arch Otolaryngol Head Neck Surg 2010;136(8):778-82. 